Archives de sciences sociales des religions

141 | janvier-mars 2008

Mémoires

\title{
L'ethnologie aux Archives : un témoignage
}

\section{Jacques Gutwirth}

\section{OpenEdition}

Journals

Édition électronique

URL : https://journals.openedition.org/assr/13122

DOI : 10.4000/assr.13122

ISSN : $1777-5825$

Éditeur

Éditions de l'EHESS

Édition imprimée

Date de publication : 1 mars 2008

Pagination : 159-165

ISBN : 978-2-7132-2189-7

ISSN : 0335-5985

\section{Référence électronique}

Jacques Gutwirth, "L'ethnologie aux Archives : un témoignage », Archives de sciences sociales des religions [En ligne], 141 | janvier-mars 2008, mis en ligne le 02 juillet 2011, consulté le 08 juillet 2021. URL : http://journals.openedition.org/assr/13122 ; DOI : https://doi.org/10.4000/assr.13122 
Jacques Gutwirth

\section{L'ethnologie aux Archives : un témoignage}

Joachim Wach, cité par André Mary dans son article «Les Archives... Cinquante ans après..." (ASSR 136, 2006), rappelle que "l'anthropologie culturelle " devait jouer un rôle important dans l'étude de la religion par les sciences sociales. Qu'en fut-il dans le développement de cette revue ? Dès le départ on trouve de nombreux articles sur le messianisme - dans deux numéros thématiques, les numéros 4 et 5 de 1957 et 1958, avec des écrits d'ethnologues, notamment ceux de Jean Guiart, Jacqueline Eberhardt, Alfred Métraux, Roger Bastide. Encore en 1965 et 1966 (19 et 21), Vittorio Lanternari traite ce thème. Il y a aussi, lors des premières années de la revue, d'autres articles de sociologues et d'historiens traitant du messianisme. À l'évidence, l'influence de Henri Desroche, alors rédacteur en chef de la revue et spécialiste (entre autres) du messianisme - sa thèse de doctorat ès lettres (1970) concerne Messianismes, utopies et sociologie des religions - joue ici son rôle. Cependant, des études d'ethnologues sur d'autres thèmes sont présentes dès le début. Ainsi en 1957 dans le numéro 3 on trouve un article de Germaine Dieterlen sur les « résultats des missions Griaule au Soudan français (1931-1956)» (1957, 3). D'autres ethnologues africanistes publient également dans la revue : René Bureau (1964, 17), Luc de Heusch (1964, 18), Ernest Gellner pour l'Afrique du Nord $(1963,15)$ sans oublier dès 1959 l'indianiste Louis Dumont $(1959,7)$ et les océanistes, avec Rosselène Dousset-Leenhardt $(1967,24)$ et encore Jean Guiart $(1968,26)$; signalons aussi un article de Roger Bastide sur « le spiritisme au Brésil » $(1967,24)$ et aussi deux articles, longs et documentés, illustrés de photos, de George Condominas en 1968 (25-26) « Notes sur le bouddhisme populaire en milieu rural lao ». Cependant, à partir de 1969 jusqu'en 1973, les études véritablement ethnologiques ou anthropologiques sont, selon mon dépouillement, passagèrement absentes ; toutefois sont présents l'islam, avec Jean-Paul Charnay (1972, 33), « Jeux de miroir... Réorientations du rapport Islam/Islamologie) » ou encore l'Afrique avec Yves Person (1973, 36), «Pour une histoire des religions africaines ", tandis que les bulletins bibliographiques comportent bien des analyses d'ouvrages ethnologiques. 


\section{L'élargissement du comité de rédaction}

Il n'est pas anodin d'examiner la constitution de la direction, puis du comité de rédaction et de lecture de la revue telle que celle-ci se présente aux lecteurs; de sa naissance en 1956, jusqu'en 1968, soit quatorze années durant, elle n'a pas officiellement de comité de rédaction, ni de comité de lecture. Mieux encore, au numéro 1, la revue est publiée collectivement par le « Groupe de sociologie des religions ». Dans plusieurs numéros du début, notamment le premier de 1956, et aussi de 1957 à 1959, on ne découvre même pas le nom de ses responsables. Néanmoins, les membres de la direction/rédaction apparaissent au numéro 2 de 1956 ; son président est Gabriel Le Bras. Au-dessous de lui sont mentionnés deux secrétaires généraux, Norman Birnbaum (sociologue, alors professeur à Londres, puis Oxford) et Henri Desroche. Et voici graphiquement (et je présume hiérarchiquement) sous eux le secrétariat/rédaction : François-André Isambert s'occupe de "mémoires et communication ", Jacques Maître de "notes et documents " et Émile Poulat du «bulletin bibliographique ». Aucun ethnologue n'est présent parmi eux. C'est seulement à partir du premier numéro de 1969 que la revue présente un comité de rédaction et un comité de lecture. Le "président " reste Gabriel Le Bras, le rédacteur en chef est désormais le seul Henri Desroche (Birnbaum ne figurant plus à ses côtés). Le comité de rédaction compte JeanPierre Deconchy, François-André Isambert, Jacques Maître, Émile Poulat et Jean Séguy. Le comité de lecture est constitué du comité de rédaction plus Jean-Paul Charnay, Jean Hadot, Marie-Louise Letendre, Jean-Marie Mayeur, Michel Meslin, Jean-Pierre Vernant, et enfin un seul ethnologue, Roger Bastide. En 1970, Gabriel Le Bras décède et à partir de 1971 la revue sera publiée, du moins officiellement, en association avec d'autres laboratoires : "le Groupe Religion et développement " représenté par Desroche, et "l'équipe de psychosociologie expérimentale des idéologies" qui est le fait du seul Jean-Pierre Deconchy. Incidemment, ce dernier assura de 1977 à 1992 des tâches importantes comme responsable du bulletin des périodiques (qui disparaît en 1992). Dans un texte signé collectivement par le comité de rédaction $(1971,31)$, il est également dit, à juste titre, que " au cours de ces quinze ans, l'éventail [de la revue] s'est déployé par les deux bouts, déploiement des domaines, déploiement des disciplines. Le premier a incorporé non seulement les religions dominantes, mais également les religions marginales... le second a proposé et accepté des rendez-vous de la sociologie avec d'autres sciences sociales... Les rapports avec l'histoire, l'ethnologie, la psychologie se sont multipliés. » (pp. 4-5). Néanmoins, à partir de 1969 seul Roger Bastide (il décède en 1974) représentait l'ethnologie à la direction de la revue.

À la suggestion de Jean Séguy (selon une communication personnelle d'Émile Poulat, confirmée par Jean Séguy), la revue modifie en 1973 son titre et élargit sa perspective en devenant Archives de Sciences sociales des religions. Jean Séguy était, je pense, particulièrement sensible à cette nécessité d'élargissement ; en effet, il s'intéressait à des phénomènes minoritaires "sectaires » (voir dès 1956 son 
livre Les sectes dans la France contemporaine et surtout en 1977 son remarquable opus magnum, Les assemblées anabaptistes-mennonites de France) ; certes il utilisait largement dans ce travail la méthode historique mais aussi l'observation participante à la manière des ethnologues, même si à cette époque il ignorait ce qu'était l'ethnologie - tel qu'il me l'a dit peu de temps avant que je n'écrive ce texte au printemps 2007, donc quelques mois avant son décès ${ }^{1}$. En tout cas le nouveau titre correspondait certainement à l'esprit de la revue et de ses animateurs, enfin aux articles publiés depuis ses débuts en 1956. Jacques Maître observe que "...l'apparition des ASR déclenchera des pressions ecclésiastiques sur Gabriel Le Bras pour qu'il noie cet enfant bâtard dont la vocation va être de promouvoir une sociologie des religions, au lieu d'une sociologie religieuse inféodée à la hiérarchie catholique... » et il ajoute : " déjà l'histoire des religions et l'ethnographie religieuse (souligné par moi) avaient largement ouvert la voie d'une liberté de pensée nouvelle à ce sujet, notamment à travers le comparatisme » (ASSR, 136 : 64). Lors de la parution en 1973 du premier numéro qui affiche le nouveau titre de la revue, dans un bref «à nos lecteurs » $(1973,35)$ signé "comité de rédaction ", il est dit : " notre changement de titre traduit un ancrage plus conscient... jusqu'ici véhiculé en filigrane, d'une science multidimensionnelle des religions » (p. 4). Certes, y lit-on encore, « disons-le bien clairement : nous n'entendons ni abandonner notre orientation principalement sociologique, ni non plus prôner une primauté de la sociologie dans la constitution d'une science générale des religions ».

En tout cas, avec un tel dessein, la place de l'ethnologie-anthropologie était évidente, sinon indispensable. D'ailleurs dès 1973 (35 janvier-juin) Louis-Vincent Thomas et moi-même devenons membres du comité de lecture de la revue. L'existence d'un comité de rédaction et d'un comité de lecture restreint subsistera jusqu'en 1980, lorsqu'une amplification pléthorique et pluridisciplinaire du comité de rédaction s'installe et persiste, avec depuis 2004 comme rédacteur en chef André Mary, un anthropologue ! L'enfant bâtard évoqué par Maître s'est désormais totalement affranchi et des autorités religieuses et de la prééminence de la sociologie; ce sont les sciences des religions qui triomphent.

\section{Ma participation aux Archives et au Groupe de Sociologie des religions}

À l'époque de mon arrivée aux Archives, j’avais été, de 1972 à 1974, professeur d'ethnologie à l'université de Provence à Aix, mais je devais revenir au CNRS à Paris en été 1974. Jacques Maître, rencontré à une manifestation politique (je

1. J'ai bien connu Jean Séguy durant mes années au Groupe de Sociologie à la rue d'Athènes, cela jusqu'en 1985 environ, mais aussi par la suite. Nous avons maintes fois dîné ensemble, conversé amicalement. C'était un grand chercheur, entièrement dévoué à ses rigoureux travaux, mais c'était aussi un homme de cœur qui respectait les groupes qu'il avait étudiés, notamment les Mennonites, bien qu'à l'évidence il ne partageât ni leur foi ni leurs idées. 
ne sais plus avec quel objectif !) me proposa d'intégrer à mon retour le Groupe de sociologie des religions : j'acceptai immédiatement. C'est aussi en 1974 que je rejoignis le comité de rédaction des Archives et j'acceptai cet honneur avec joie. Je siégeai au comité de rédaction, alors très réduit. Je me souviens très bien des premières réunions auxquelles j'ai assisté avenue Franco-russe, dans un petit bureau, celui de Henri Desroche je crois, nous étions en général cinq présents : Émile Poulat, Jean Séguy, François-André Isambert, Jacques Maître et moi ; quant à Desroche, il participait par intermittence, occupé à d'autres activités dans une pièce voisine. J'étais désormais le représentant de l'ethnologie à ce comité. Certes, j'étais un ethnologue un peu particulier puisque mon objet de recherche "exotique ", le hassidisme judaïque, se trouvait dans une grande ville à 350 kilomètres de Paris; d'autre part, avec ma spécialité, je représentais aussi le judaïsme.

En tout cas le comité avec ma présence, accentuait le caractère pluridisciplinaire de la revue. Il me semble que je restai le seul ethnologue au comité de rédaction proprement dit jusqu'à l'élargissement de 1980. Comme membre du comité de rédaction, je m'efforçai de susciter la parution d'articles d'ethnologues. À mon invitation, de jeunes anthropologues africanistes, que je connaissais bien personnellement, apportèrent des articles à la revue : Jean-Claude Muller avec "Old Wine in New Wineskins. Traditionalists and Christians among the Rukuba " (Vin vieux, outres neuves. Traditionalistes et chrétiens chez les Rukuba) $(1974,38)$ et Nicole Echard avec « Petites lectures sur des phénomènes de possession africains » $(1975,40)$. Dans le numéro 38 de 1974 parut aussi un article de Claudine Vidal, «De la religion subie au modernisme refusé : "théophagie”, ancêtres clandestins et résistance populaire au Rwanda". Puis il y aura des contributions de Rosselène Dousset-Leenhardt, « À propos du totémisme autour de la Mer de Corail» (1975, 40). La même année, parut un article de Martine Segalen, ethnologue de la France, sur « rites funéraires en Normandie » (1975, 39). Parurent encore "Christianisme, Islam et transformations sociales. La famille en Casamance » de Jacqueline Trincaz, et "Comment être femme. Un aspect du rituel Mukisi chez les Téké de la République populaire du Congo » de Marie-Claude Dupré, tous deux dans le même numéro (1978, 46-1). Je lus plusieurs de ces articles pour remarques et commentaires avant leur acceptation.

Dès mon arrivée au GSR, Émile Poulat, son dynamique directeur, me demanda d'y créer une équipe "ethnologie », qui fut mise en place en 1974 : elle comprenait Constant Hamès (Afrique Blanche), René Luneau (Afrique noire) et, en 1978, trois chercheurs associés, Rosselène Dousset-Leenhardt (NouvelleCalédonie), Annie Comolli, (alors notamment cinéaste des rites judaïques), Jacqueline Trincaz, africaniste, et moi-même. Cette équipe se réunit assez régulièrement pendant plusieurs années - sous la direction de René Luneau pendant ma mission d'un an aux États-Unis en 1975-76 - mais des raisons diverses, notamment l'absence d'insertion professionnelle stable comme chercheur ou 
universitaire de la plupart de ses membres, empêchèrent à cette époque un véritable développement de cette équipe.

Personnellement, j'apportai dans la revue, dès 1975 (40, 1), un regard ethnologique sur nos propres sociétés avec un premier article comparant deux groupes religieux «piétistes » - mot que j'empruntai à Émile Poulat -, les hassidim et une église évangélique en Cévennes En 1979 (47-2), j’ouvris une nouvelle piste, celle de l'analyse prompte d'un phénomène religieux sensationnel tout récent, avec mon article sur « le suicide-massacre de Guyana et son contexte ». Mais la piste de l'examen de l'événement immédiat, ne fut pas, me semble-t-il, à l'époque véritablement poursuivie à la revue.

En 1988 je quittai définitivement le Groupe de Sociologie pour diriger, avec mon amie Colette Pétonnet, le Laboratoire d'anthropologie urbaine du CNRS (créé par nous en 1985 en tant que "Jeune équipe ») ; j'ai néanmoins organisé, en 1991, en quelque sorte avec ma double casquette d'ethnologue « urbain » et " religieux ", un numéro thématique " anthropologie urbaine religieuse » (73) dont les contributeurs (hormis Jean-Marie Gibbal et moi-même) avaient rencontré le fait religieux à travers des sujets d'études autres. Je montrai dans mon introduction à ce numéro les atouts et les handicaps de cette situation. J'écrivais : "d'une part le chercheur qui rencontre secondairement un fait religieux déterminé - tel Patrick Williams découvrant peu à peu l'importance du pentecôtisme chez les tsiganes - peut mieux le situer dans la globalité des phénomènes socioculturels qu'il connaît bien; d'autre part, ce chercheur - P.W. ou un autre - est a priori moins connaisseur des religions traitées qu'un sociologue ou historien spécialiste de telle ou telle religion qui lui, il est vrai sera peut-être moins expert quant aux tenants et aboutissants divers - le contexte global » (p. 6). En tout cas, ce numéro a introduit aux Archives d'excellents anthropologues : outre Patrick Williams, notamment Catherine Choron-Baix qui traita de la transplantation du bouddhisme lao en France, Anne Raulin et Jeanne Brody qui examinèrent « la dimension esthétique et sacrée de l'écologie urbaine. La Petite Asie à Paris » (dans le $13^{\mathrm{e}}$ arrondissement), Jeanine Fribourg qui présenta les rues de la ville en Espagne comme scène du religieux (avec les processions, pasos, de la Semaine sainte) et Sylvie Fainzang qui traita des textes de suppliques de guérison à Notre Dame de la Garde à Marseille. D'autre part, Jean-Marie Gibbal (hélas trop tôt décédé en 1993) offrait une belle description d'un phénomène de possession très mondain, relevant des religions afro-brésiliennes, Claudia Fonseca (professeur au Brésil) examinait la religion populaire, très syncrétiquement vécue par des milieux populaires de bidonvilles, tandis que je traitais du pentecôtisme et d'audiovisuel ; or ces trois contributions avaient comme cadre la ville de Porto Alegre (nous participions à un programme d'échange scientifique et universitaire francobrésilien dans cette ville).

Les Archives ont quasiment dès le départ entretenu un lien privilégié avec le Brésil, ses chercheurs et ses diverses religions. Roger Bastide en fut en quelque 
sorte l'intercesseur privilégié et moi-même, qui avais vécu à Rio à la même époque, mais en tant que lycéen, je poursuivais ainsi cette vocation particulière de la revue. Dès 1977, j'avais consacré une note de lecture à des livres brésiliens intéressants, à propos de divers lieux de pèlerinage : "Aspects du catholicisme au Brésil »(44-2).

En 1993, dans le numéro (83) consacré à la religion aux États-Unis, j’ai présenté un article "Religion télévisée et business audio-visuel », partant de mon expérience d'anthropologue de terrain pour en arriver, grâce à l'étude d'un certain nombre de documents (à ce moment encore sans l'apport inestimable des moteurs de recherche et des sites web sur internet), à examiner les liens ahurissants entre leaders " religieux » et affairisme. Les plus importants télévangélistes/ hommes d'affaires, étaient, et sont encore, Pat Robertson - qui s'est même voulu candidat à la présidence des États-Unis au sein du parti républicain en 1988 et Paul Crouch, animateur toujours actif d'une chaîne télévisuelle sur satellite (Trinity Broadcasting Network). Citons aussi Jim Bakker qui finit en prison après des affaires frauduleuses, ou Jerry Falwell, fondateur d'une "majorité morale » très politique et entrepreneur télévisuel plutôt raté.

J'ai donc appartenu au Groupe de sociologie des religions de 1974 jusqu'en 1985 et je suis resté au comité de rédaction des Archives pendant trente ans, jusqu'en 2004, y soutenant l'apport de l'ethnologie sous toutes ses facettes. Naturellement, j’ai aussi participé au dépouillement des périodiques des Archives, avec notamment, mais pas uniquement, l'examen de revues d'ethnologie, ainsi qu'au bulletin bibliographique, avec des comptes rendus d'ouvrages d'ethnologues.

Ainsi donc sans exagérer la portée de ma présence aux Archives et au Groupe de Sociologie des Religions, je pense qu'elle a quelque peu concouru à la présence de l'ethnologie-anthropologie.

Jacques GUTWIRTH jgutwirth@aol.com

BASTIDE Roger, 1958, "Le messianisme raté », ASR, 5, pp. 31-37.

-, 1967, «Le spiritisme au Brésil », ASR, 24, pp. 3-16.

Bureau René, 1964, "Flux et reflux de la christianisation camerounaise », ASR, 17, pp. 97-112.

Charnay Jean-Paul, 1972, " Jeux de miroir et crise de civilisations. Réorientations du rapport Islam/islamologie », ASR, $33: 135-174$.

Comité de rédaction, 1973, "À nos lecteurs ", ASSR, 35, pp. 3-5.

CONDOMINAS Georges, 1968, "Notes sur le bouddhisme populaire en milieu rural lao ", ASR, 25, pp. 81-116.

-, 1968, "Notes sur le bouddhisme populaire en milieu rural lao (II) », ASR, 26, pp. 116-145. 
Dieterlen Germaine, 1957, «Les résultats des missions Griaule au Soudan français (1931-1956) », ASR, 3-1, pp. 137-142.

Dousset-LeEnHARdT Rosselène, 1967, «En Nouvelle-Calédonie. À propos de kamo cynomorphes ", ASR, 24, pp. 135-139.

-, 1975, «À propos du totémisme autour de la Mer de Corail », ASSR, 40, pp. 107-117. DumONT Louis, 1959, "Le renoncement dans les religions de l'Inde », ASR, 7, pp. 45-69.

Dupré Marie-Claude, 1978, "Comment être femme. Un aspect du rituel Mukisi chez les Téké de la République populaire du Congo », ASSR, 46-1, pp. 57-84.

Eberhardt Jacqueline, 1957, "Messianisme en Afrique du Sud », ASR, 4, pp. 31-56.

ECHARD Nicole, 1975, "Petites lectures sur des phénomènes de possession africains ", ASSR, 40, pp. 145-156.

Gellner Ernest, 1963, "Sanctity, Puritanism, Secularisation and Nationalism in North Africa ", ASR, 15, pp. 71-86.

GUIART Jean, 1957, «Institutions religieuses traditionnelles et messianismes modernes à Fiji », ASR, 4, pp. 3-30.

-, 1968, «Des multiples niveaux de signification du mythe » ASR, 26, pp. 55-71.

GUTWIRTH Jacques, 1975, "Piétistes juifs et protestants. Une analyse comparative ", ASSR, 40, pp. 53-66.

-, 1977, "Aspects du catholicisme au Brésil », ASSR, 44-2, pp. 175-180.

-, 1979, «Le suicide-massacre de Guyana et son contexte », ASSR, 47-2, pp. 167-187.

-, 1991, "Anthropologie urbaine religieuse : une introduction », ASSR, 73, pp. 5-15.

-, 1993, "Religion télévisée et business audio-visuel ", ASSR, 93, pp. 67-89.

LANTERNARI Vittorio, 1965, "Syncrétismes, messianismes, néo-traditionalismes en Afrique noire », ASR, 19, pp. 99-116.

-, 1966, "Syncrétismes, messianismes, néo-traditionalismes en Afrique noire » (II), ASR, 21, pp. 101-110.

Comité de rédaction, 1971, «Du Groupe de Sociologie des Religions à un Institut de Sciences Sociales des Religions ", ASR, 31, pp. 3-6.

MAîTRE Jacques, 2006, "Ruminations d'un dinosaure ", ASSR, 136, pp. 63-65.

MARY André, 2006, "Les Archives... Cinquante ans... après ", ASSR, 136, pp. 9-24.

Metraux Alfred, 1957, «Les Messies de l'Amérique du Sud », ASR, 4, pp. 108-112.

Muller Jean-Claude, 1974, « Old Wine in New Wineskins. Traditionalists and Christians among the Rukuba/Vin vieux, outres neuves. Traditionalistes et chrétiens chez les Rukuba ", ASSR, 38, pp. 49-62.

Person Yves, 1973, "Pour une histoire des religions africaines ", ASR, 36, pp. 91-101.

Segalen Martine, 1975, «Rites funéraires en Normandie ", ASSR, 39, pp. 79-88.

SÉGUY Jean, 1956, Les sectes protestantes dans la France contemporaine, Paris, Bauchesne (réédition 1997).

-, 1977, Les assemblées anabaptistes-mennonites de France, Paris, La Haye, Mouton.

TrincAZ Jacqueline, 1978, "Christianisme, Islam et transformations sociales. La famille en Casamance ", ASSR, 46-1, pp. 85-109.

VIDAL Claudine, 1974, «De la religion subie au modernisme refusé: "théophagie", ancêtres clandestins et résistance populaire au Rwanda », ASSR, 38, pp. 63-90. 
